\title{
The effect of a live-high/train-high regimen on emotional state
}

\author{
Adam C McDonnell ${ }^{1,2^{*}}$, Nektarios AM Stavrou ${ }^{3,4}$, Ola Eiken ${ }^{5}$, Igor B Mekjavic ${ }^{1}$ \\ From 15th International Conference on Environmental Ergonomics (ICEE XV) \\ Portsmouth, UK. 28 June - 3 July 2015
}

\begin{abstract}
Introduction
We have previously reported [1] that 10 day hypoxic bedrest induces psychological strain, which is not evident during normoxic bedrest. In addition, daily ambulation while confined to a hypoxic environment also appears to prevent the hypoxic inactivity induced psychological strain. In view of the growing popularity of hypoxic training, particularly among winter athletes who live and train at altitude (Live-High/Train-High, LH/TH), we investigated the effect of such a training regimen on emotional state, as well as on the interaction among the psychological indices.
\end{abstract}

\section{Methods}

Fourteen male participants took part in a 10-d confinement to normobaric hypoxia $\left(\mathrm{P}_{\mathrm{I}} \mathrm{O}_{2}=88.2 \pm 0.6 \mathrm{mmHg}\right.$; simulated altitude of $4175 \mathrm{~m}$ ), conducted at the Olympic Sport Centre Planica (Rateče, Slovenia). The participants were randomly assigned either to a Live-High/Train-High group (LH/TH: two 60-minute moderate intensity exercise sessions daily on a cycle ergometer), or to a Live-High (LH) group. The participants completed the Profile of Mood States (POMS) and the Positive Affect and Negative Affect Schedule (PANAS) instruments, two days before the onset of the confinement (PRE), on the $3^{\text {rd }}$ (D3), $7^{\text {th }}(\mathrm{D} 7)$ and $10^{\text {th }}(\mathrm{D} 10)$ day of the confinement and on the second day of recovery (POST).

\section{Results}

There were no significant differences revealed across the five measures in the POMS or PANAS subscales in either group (LH/TH: $\mathrm{p}=0.325$ to $0.788, \mathrm{LH}: \mathrm{p}=0.345$ to 0.760$)$. High positive correlations were revealed among the negative moods (Depression, Anger, Confusion, Fatigue and Tension) during hypoxic confinement (D3: $r_{\text {mean }}=0.88, D 7: r_{\text {mean }}=0.81$, D10: $\left.r_{\text {mean }}=0.60\right)$, while low to medium negative correlations were revealed between the positive and negative psychological indices.

\section{Conclusion}

Hypoxic confinement induces a high correlation among the negative POMS subscales and depression. Increasing the level of daily exercise to moderate does not ameliorate this negative psychological profile.

\section{Acknowledgements}

The research leading to these results has received funding, in part, from the European Union's Framework Programme (2007-2013) under grant agreement no. 284438 (project PlanHab: Planetary Habitat Simulation) and the Slovene Research Agency.

\section{Authors' details}

'Department of Automation, Biocybernetics and Robotics, Jozef Stefan Institute, Jamova Cesta 39, 1000 Ljubljana, Slovenia. ${ }^{2}$ Jozef Stefan International Postgraduate School, Jamova Cesta 39, 1000 Ljubljana, Slovenia. ${ }^{3}$ Aspetar, Orthopaedic and Sports Medicine Hospital, Doha, Qatar. ${ }^{4}$ National \& Kapodistrian University of Athens, Athens, Greece. ${ }^{5}$ Department of Environmental Physiology, Royal Institute of Technology, Stockholm, Sweden.

Published: 14 September 2015

\section{Reference}

1. Stavrou NA, McDonnell AC, Eiken O, Mekjavic IB: Psychological strain: Examining the effect of hypoxic bedrest and confinement. Physiol Behav 2015, 139:497-504.

\section{doi:10.1186/2046-7648-4-S1-A54}

Cite this article as: McDonnell et al:: The effect of a live-high/train-high regimen on emotional state. Extreme Physiology \& Medicine 2015 4(Suppl 1):A54.

\footnotetext{
* Correspondence: adam.mcdonnell@ijs.si

'Department of Automation, Biocybernetics and Robotics, Jozef Stefan

Institute, Jamova Cesta 39, 1000 Ljubljana, Slovenia

Full list of author information is available at the end of the article
} 